\title{
AXIAL FLUX PROFILE IN THE ADVANCED TEST REACTOR
}

\author{
Nathan Manwaring ${ }^{1,2}$ and Michael Reichenberger ${ }^{1}$ \\ ${ }^{1}$ Idaho National Laboratory \\ 2525 Fremont, Idaho Falls, ID 83415 \\ ${ }^{2}$ University of Idaho \\ 1776 Science Center Drive, Idaho Falls, ID 83402 \\ Nathan.Manwaring@inl.gov, Michael.Reichenberger@inl.gov
}

\begin{abstract}
This work demonstrates an approach to determine probability of perturbation of the axial profile of the thermal neutron flux in the Advanced Test Reactor. The axial flux profile is expected to follow a theoretical cosine shape, due to the minimal use of vertically-withdrawn shims. Reactivity is normally controlled by rotation of Outer Shim Control Cylinders, uniformly affecting neutron flux at all axial locations. The Advanced Test Reactor routinely accepts for irradiation experiments of a variety of designs. Among the analyses required by the safety basis approved by the United States Department of Energy is the characterization of a new experiment's potential for perturbing the axial flux, which could exacerbate power peaking in the driver fuel. However, this perturbation can be more or less severe in different locations within the fuel. Therefore, the best characterization of axial flux perturbation requires knowledge of baseline axial flux. Such information is obtained by measuring decay in activated uranium flux wires irradiated at known positions in cooling channels in plate-type fuel elements. Due to variability in measured axial flux, it is not usually clear whether a given anomalous measurement is caused by an actual perturbation. Assuming normality in random measurement errors, the probability of an actual perturbation is quantified.
\end{abstract}

KEYWORDS: Advanced Test Reactor, ATR, Axial, Probability

\section{INTRODUCTION}

The ATR core (see Figure 1) consists of forty (40) plate-type fuel elements, of aluminum-clad highlyenriched uranium. Pressurized water is both the moderator and the primary coolant and flows downward through the fuel element channels. The forty elements are organized around nine (9) flux traps, and power is shifted radially and azimuthally by means of control elements such as outer shim control cylinders, in order to obtain desired powers for irradiation of flux trap experiments. The purpose of localized power control is to simultaneously irradiate multiple experiments in the various flux traps, at programmable power levels. Most of the flux traps include the additional advantage of an in-pile tube, isolated from the reactor primary coolant system, which allows a given experiment to be irradiated at temperature, pressure, and chemistry conditions selected by the sponsor. 


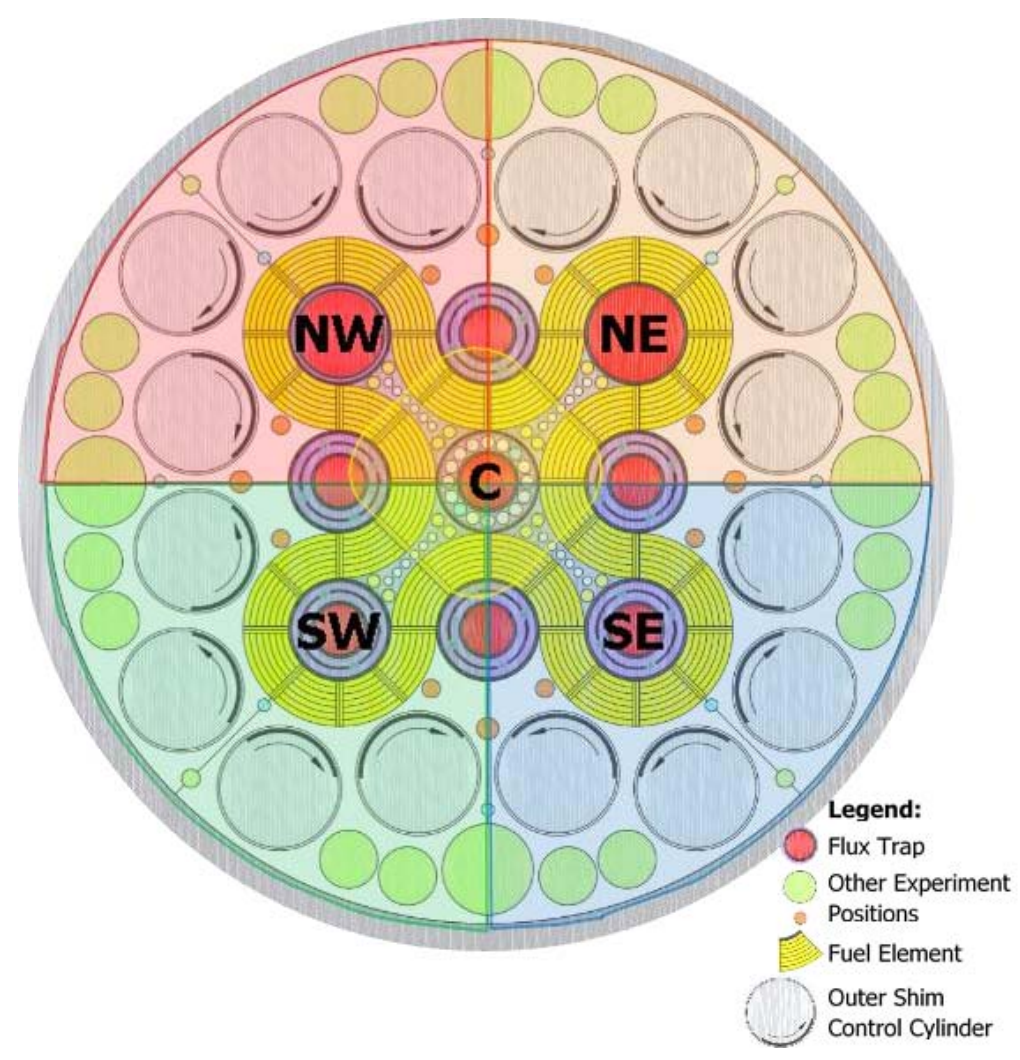

Figure 1. Plan View of ATR Core.

For convenience, fuel elements are grouped into five lobes, named for the Center (C) and for the cardinal direction of each corner (Northwest (NW), Northeast (NE), Southwest (SW), and Southeast (SE)). The C lobe is in some contexts divided into fourths, allowing the ATR core to be grouped into four quadrants (NW, NE, SW, and SE). Each lobe contains a flux trap. The four other flux traps are known as outer flux traps, for being outside the closed fuel serpentine, and are designated by cardinal directions (North $(\mathrm{N})$, West (W), East (E), and South (S)). Power is said to be produced by each lobe or quadrant and is indicated to operators. Outer flux trap powers are also indicated, as the average of the C lobe and the two adjacent corner lobes.

As indicated in Figure 1, Outer Shim Control Cylinders are withdrawn by rotating such that the hafnium plate is moved closer to or further from the fuel. In addition to providing localized control for each corner lobe, the axial shape of the neutron flux is largely unperturbed.

Prior to irradiation in ATR, experiments are measured in the companion ATR Critical facility (ATRC). ATRC is a pool-type reactor of the same size as ATR. Reactivity impacts of various experiments can be determined, usually for validation of models. ATRC has been used extensively at Idaho National Lab and has also been used for published benchmarks [1]. The axial flux profile can also be measured, as described below. By taking these measurements in ATRC, it can be shown what effect a given experiment will have on ATR.

\section{THEORY}

The ATR or ATRC core is contained within a right circular cylinder. Due to leakage, the axial flux will take a basic cosine shape. Any measurement of flux at a given axial height will have a normal probability distribution, centered about the true value. 


\subsection{Unperturbed Axial Profile}

The ATR safety basis requires a certain margin to critical heat flux, which is verified by calculating the amount of subcooling at each axial elevation in the hottest coolant channel. When the peak fission rate is shifted from the core centerline or when heat flux is too high at a given location, assumptions regarding the coolant flow and heat transfer regimes can be challenged. Furthermore, verifying an unperturbed axial flux profile ensures that fuel elements will deplete evenly, avoiding the formation of localized pockets of high fission density. For each new experiment destined for ATR, representative measurements can be made in ATRC to characterize the axial flux profile in adjacent fuel elements. The unperturbed fission profile in ATR or ATRC is described by Equation 1, shown graphically in Figure 2.

$$
F_{a}(z)=1.41 \cdot \cos (0.05775 \cdot z),
$$

$F_{a}$ in Equation 1 is the axial peaking factor: the ratio of the flux (or the fission rate) at elevation $z$ inches to the channel average. The height of the active region of ATR fuel is 48 ".

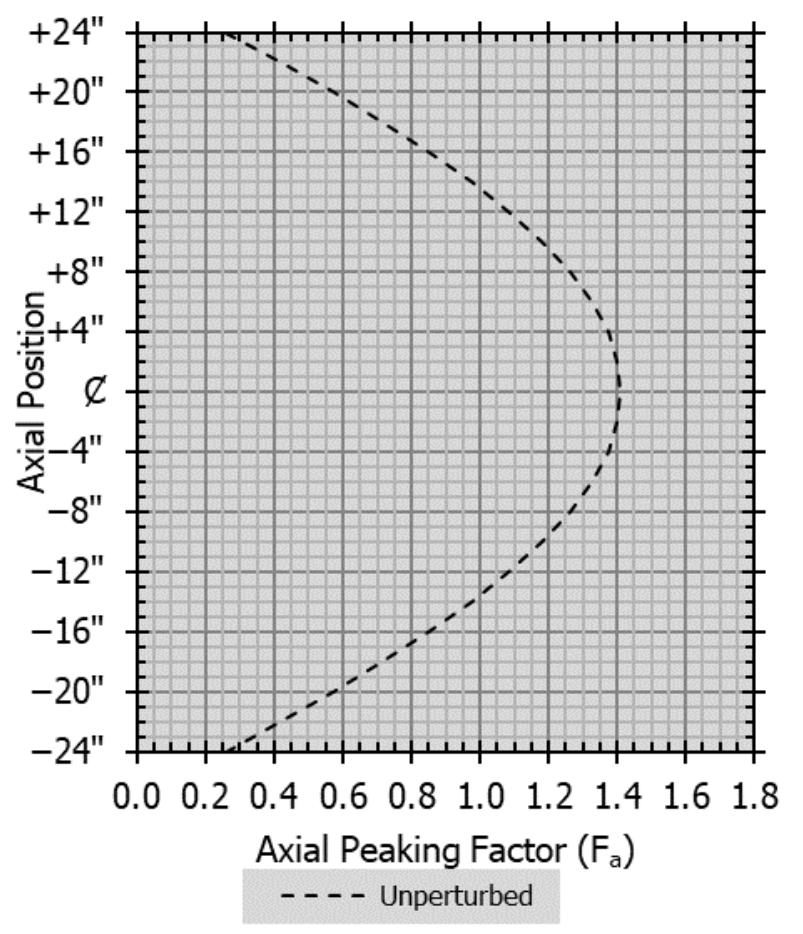

Figure 2. Unperturbed Axial Profile.

\subsection{Probability Distribution of Measurements}

Measurements of fission rate are assumed to be random variables, distributed normally about the true value. This use of the normal distribution dates to the distribution's initial formulation by Gauss [3]. Figure 3 shows a theoretical probability distribution function and cumulative distribution function. 


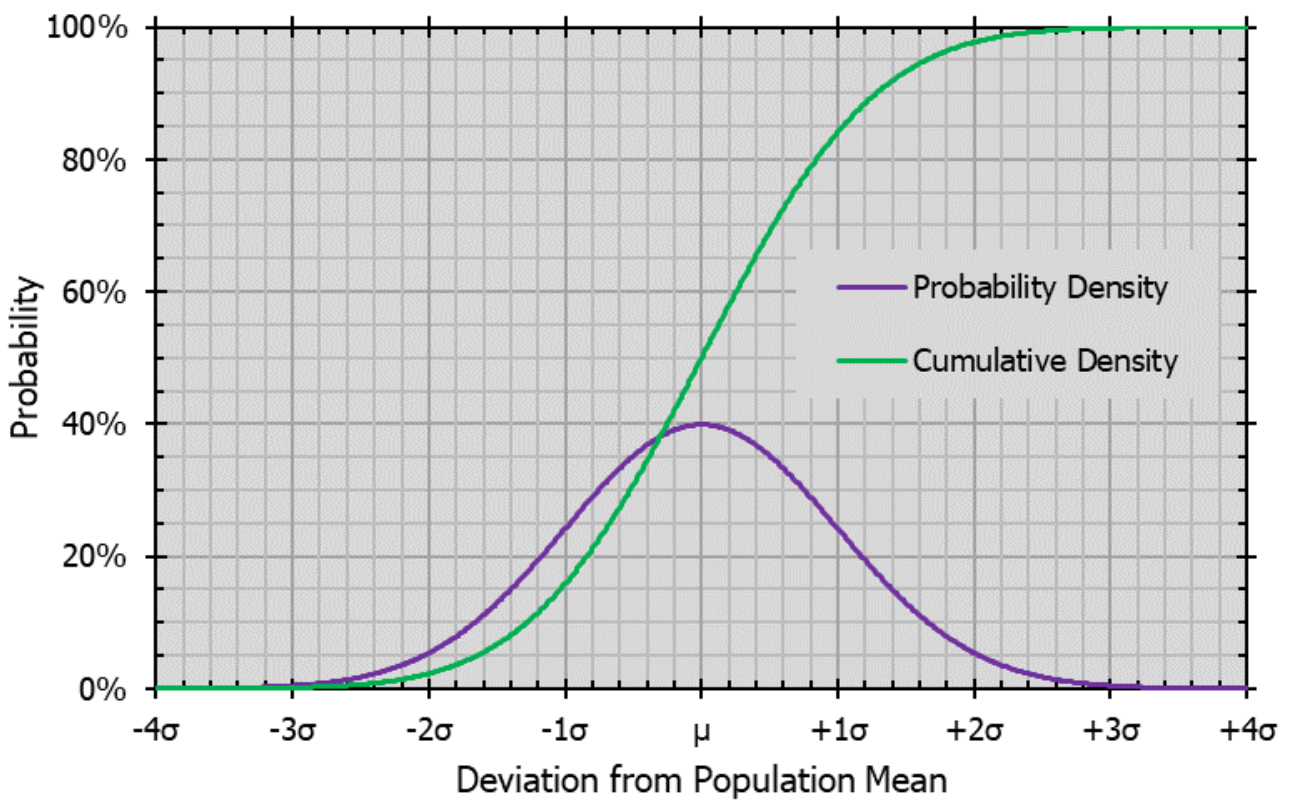

Figure 3. Normal Distribution.

With sufficient probability that the true value of the heat generation rate is higher than analyzed for the amount of subcooling, the need for additional thermal-hydraulic analyses is indicated. This must be taken with the perspective that if the results of the measurement are exactly the unperturbed profile, there is $50 \%$ probability that the true fission rate at a given height is above the measurement. With some knowledge of the standard error in the measurement process, the normal distribution can be used to find the probability that the true fission rate is above an arbitrary measurement. To decrease this probability, additional measurements must be taken at each height and show lower fission rates.

\subsection{Propagation of Uncertainty}

Uncertainties in measured fission rate at various locations all impact the computation of axial peaking factor $F_{a}$. For each parameter computed in this study, the standard error is assumed to be a combination of independent, random errors in the input parameters. Therefore, the square of the combined standard error is assumed to be the sum of the square standard errors, scaled by their effect on the computed parameter, according to Equation 2.

$$
\left.\sigma_{f\left(x_{1}, x_{2}, x_{3}, \ldots\right)}\right)^{2}=\sum_{i}\left(\sigma_{x_{i}} \cdot \frac{d}{d x_{i}}\left[f\left(x_{1}, x_{2}, x_{3}, \ldots\right)\right]\right)^{2}
$$

\subsection{Comparison to Unperturbed Profile}

As the ATR safety basis requires that the axial peaking factor be determined for each of 5 axial nodes at which thermal safety margins have been characterized. The cumulative sum of power fractions must also be determined for each of these nodes. Both calculated parameters are required to be conservatively lower than established criteria. Assuming that measurements are independent of each other, this is essentially a 10 -stage experiment, because a measured flux profile may be shown to fail either of the two criteria at any one or more nodes. 
If a measurement of a profile exactly matched the unperturbed case, the probability that it is actually perturbed in the non-conservative direction is the complement of the probability of not being perturbed in all 10 experiment stages: $1-\frac{1}{2^{10}}=99.9 \%$.

\section{METHODS}

The axial flux profile in ATR and ATRC is characterized routinely, as follows.

\subsection{Monte Carlo Simulation}

Nearly all new experiments are analyzed using some Monte Carlo method prior to irradiation in ATR. In general, this modeling work takes place prior to measurement in ATRC; therefore, ATRC measurements are used to validate models.

\subsection{Axial Profile Measurement}

Flux wands are loaded into ATRC fuel elements, with Uranium-235 $\left({ }^{235} \mathrm{U}\right)$-bearing flux wires distributed at each 2" of height [4]. Use of ${ }^{235} \mathrm{U}$ flux wires is described in [5]. After 20 minutes of irradiation at about 600 watts (W) total core power, the flux wands are removed, and decays in the flux wires are counted. Obtaining this data by irradiating flux wires is performed in ATRC approximately four or five times per calendar year, depending on experiment needs. For ATR, this process is done only upon a major core reconfiguration; therefore, it is done as a set of three or so measurements approximately every ten years. A sample of such measurements in ATRC is presented in 4. RESULTS.

The present work discusses only the probability that the axial fission profile is perturbed in a nonconservative way, not addressing whether a perturbation is beyond the safety basis.

\subsection{Uncertainty in Flux Wire Counting}

Uncertainty in flux-wire counting results is computed as the standard error of a set of counting data of a single core configuration. The most commonly used core loading in ATRC is core loading 12-13, which was first established in 2012. Power distribution has been measured in this core loading seven times, once each year 2013-2019 for annual calibration of nuclear instruments. Thus a set of seven measurements exists for each flux wire position. Three hundred forty positions (20 elements measured $\times 17$ positions at core midplane) were measured in each of these flux runs, resulting in 340 standard deviation calculations. These standard deviations are independent estimates of the true standard error of the measurement method at each location.

\section{RESULTS}

The following results were obtaining, regarding uncertainty in the measurement process and implications on completed axial measurements.

\subsection{Uncertainty in Flux Wire Counting}

Each power distribution measurement requires a flux run, and counting of flux wires reveals that the total core power during these flux runs was near the intended $600 \mathrm{~W}$ but varied by as much as $25 \%$. The standard deviation of measurements of each of 340 flux wires are illustrated in Figure 4.

Each standard deviation shown in Figure 4 is taken as an estimate of the true uncertainty of the measurement process. The set of 340 measurements is characterized by an average $\left(9.808 \times 10^{7}\right)$ and a standard deviation $\left(3.677 \times 10^{7}\right)$. 
$1.586 \times 10^{8}$ is 1.645 standard deviations above the average and is therefore the value below which $95 \%$ of randomly varying sample standard deviations will be found. However, any assumed standard error will affect all propagated uncertainties approximately equally. Therefore, the qualitative conclusion on this present work is not impacted by uncertainty in this standard error.

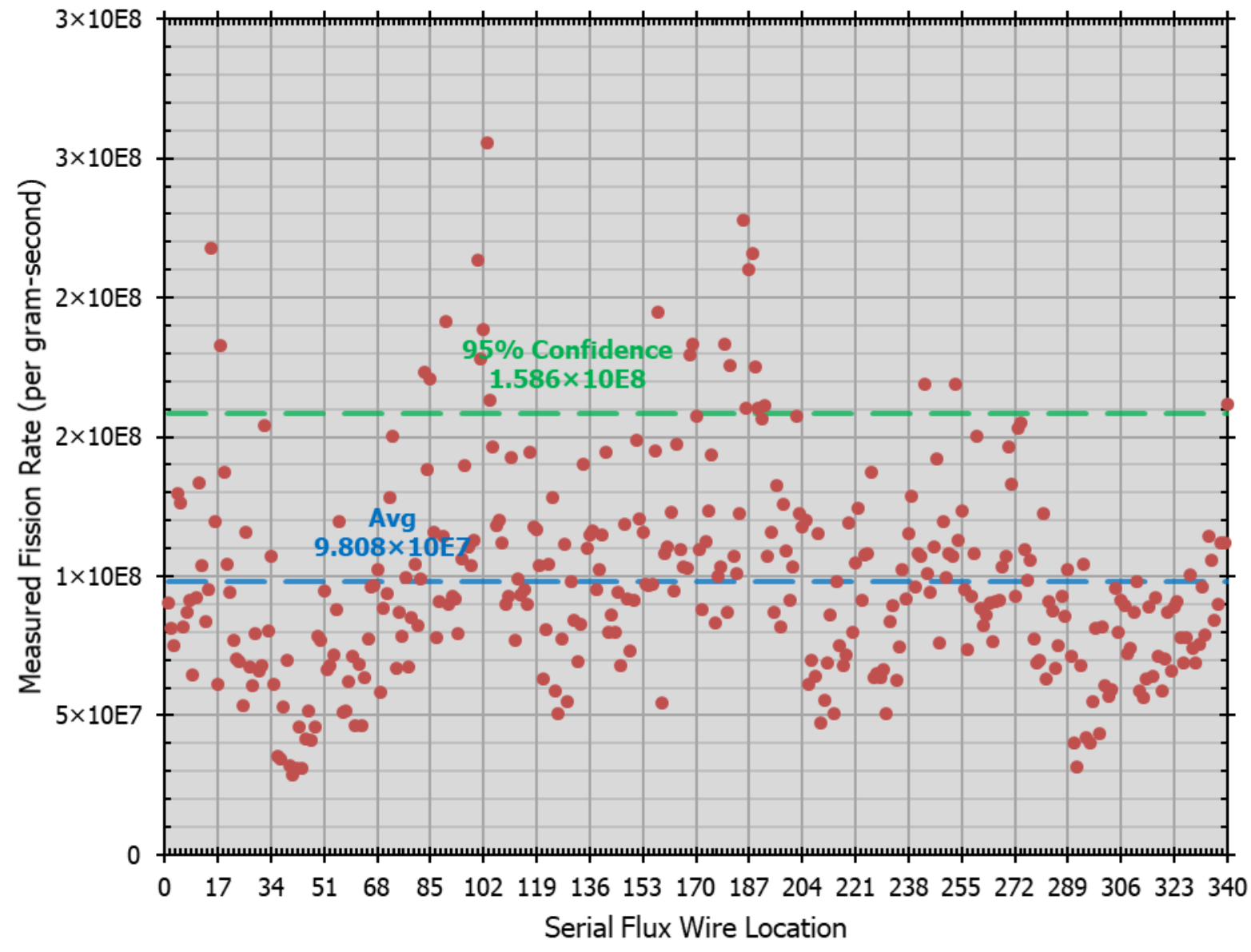

Figure 4. Standard Deviations of Fission Rate Measurements.

\subsection{Implication for Analyzed Axial Fission Profiles}

Two hundred forty-eight axial profiles were analyzed, a few of which are shown in Figure 5, with comparison to the unperturbed profile shown in Figure 2. Profiles selected for Figure 5 are those with higher 
perturbation. The theoretical unperturbed profile is shown in a bold black dashed line; individual analyzed profiles are shown in various colors.

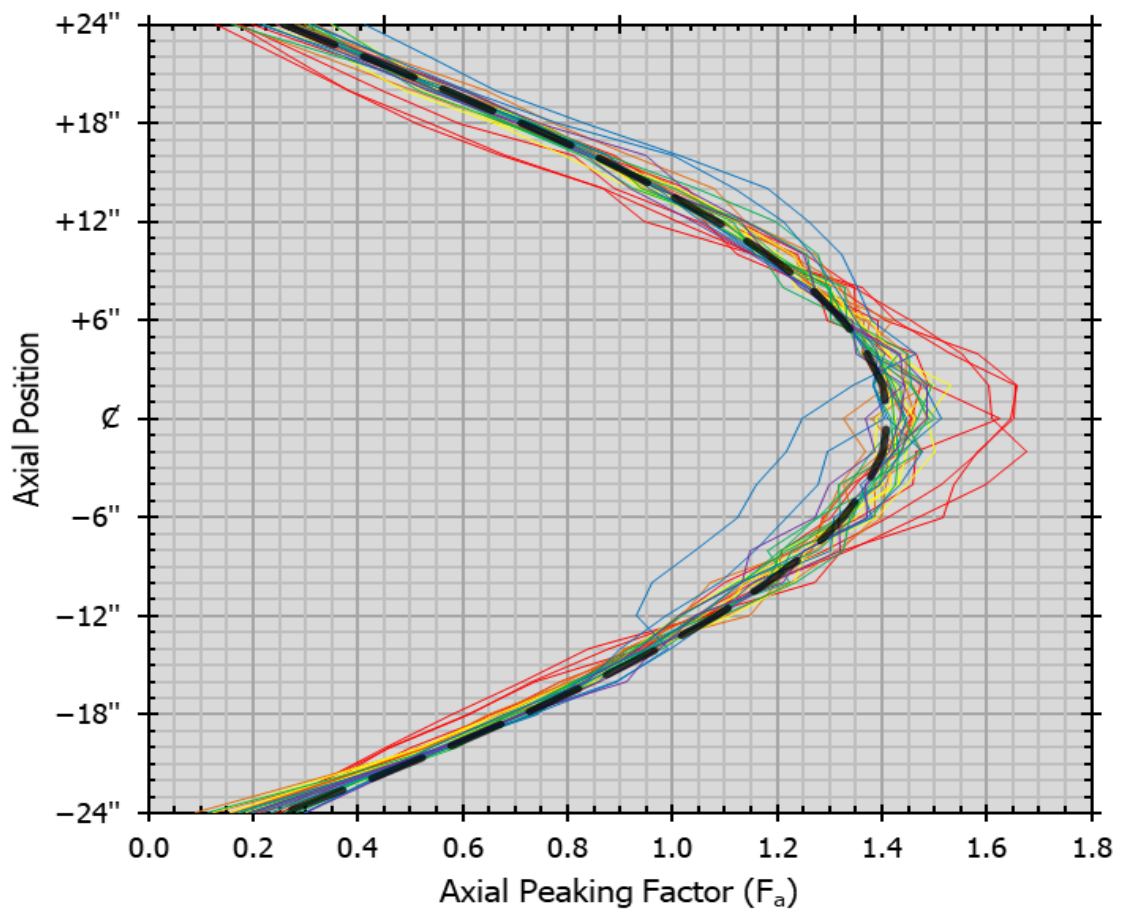

Figure 5. Selected Axial Profiles with Higher Perturbation.

Figure 6 shows a relative probability for all two hundred forty-eight profiles evaluated for this work. The probability of perturbation in the true profile giving rise to each measurement is assessed relative to the probability that a profile is perturbed when measured as exactly the unperturbed profile in Equation 1. The 
standard error from section 4.1 is propagated from each measured axial point, as described in Section 2.3, to determine the standard deviation used for comparison with the normal distribution shown in Figure 3.

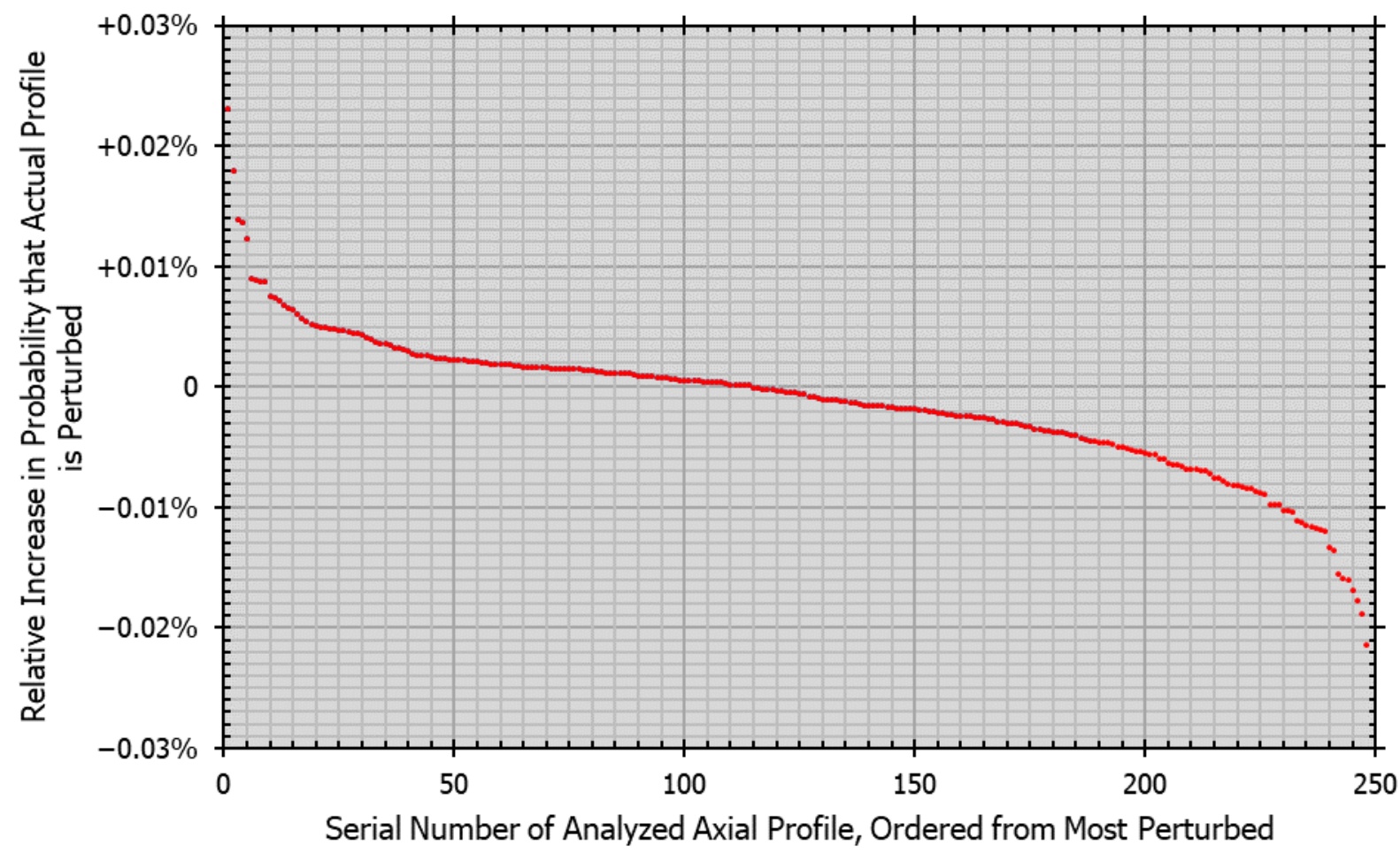

Figure 6. Probability of a True Perturbation in Each Evaluated Axial Profile.

If a given profile measurement exactly matched the unperturbed profile, it would indicate a $0 \%$ increase in relative probability that the actual fission profile is perturbed high (non-conservatively). It is seen in Figure 6 that some of the measured profiles are actually less probable to be perturbed than is one exactly matching the unperturbed profile. This is situation is possible whenever a given measurement shows a large number of data points with fission rates below the unperturbed profile.

\subsection{Monte Carlo Simulations}

The experiments that gave rise to the ATRC axial fission profiles shown as perturbed in Figure 6 were modeled prior to insertion in ATRC. Results from ATRC confirmed a priori calculations, except in a few cases where the measurement data is suspected of error. Figure 7 shows an example erroneous measurement. 


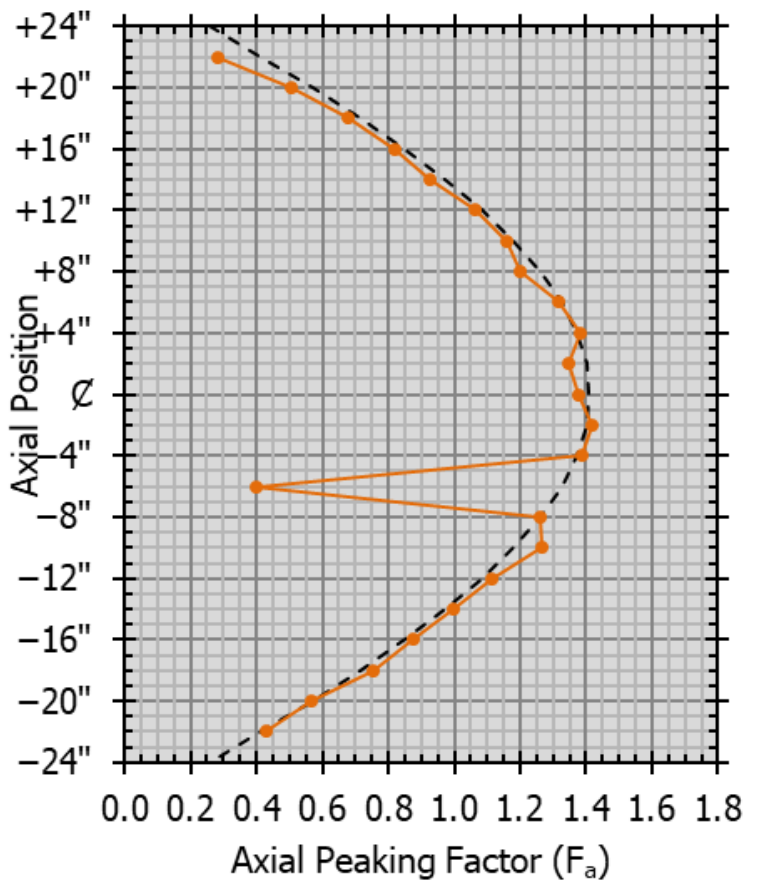

Figure 7. Example Erroneous Profile Measurement.

Profiles numbered 1 through 4 and 6 in Figure 6 are from an experiment previously reported by Nielsen [2] and are examples where measurement validated Monte Carlo calculations.

\section{CONCLUSIONS}

Using basic principles of probability theory, ATRC measurements of flux profiles can be rigorously compared against the ATR safety basis, and the probability of perturbation can be meaningfully quantified. Outliers in probability are easily observed and can be quantitatively compared.

\section{REFERENCES}

1. J. D. BESS, D. BELLER, and F. HUA, "Development and Validation of a Nuclear Criticality Benchmark Capability in the Advanced Test Reactor Critical," Trans. Am. Nucl. Soc., 109, 834 (2013).

2. J. W. NIELSEN, D. W. NIGG, and D. R. NORMAN, "Extension of the Advanced Test Reactor Operating Envelope Via Enhanced Reactor Physics Validation Techniques," Nuclear Technology, 201, 228 (2018).

3. F. J. SAMANIEGO, Stochastic Modeling and Mathematical Statistics, 141, CRC Press, Boca Raton, Florida, United States (2014).

4. M. A. REICHENBERGER, et al, "Comparison of fluence-rate determination techniques for the Advanced Test Reactor-Critical Facility," International Conference on Advancements in Nuclear Instrumentation Measurement Methods and their Applications, Portorož, Slovenia, June 17-21, 2019.

5. B. W. HOWES, C. H. HOGG and L. D. WEBER, "Use of U-235 Wire as a Fission Rate and Thermal Flux Monitor PTR-759," National Reactor Testing Station, US Atomic Energy Commission, Idaho Falls, ID, 1965. 